\title{
Improvement of Shaped Charge Penetration Capability and Disturbation of the Jet by Explosive Reactive Armor
}

\author{
Hicham KEMMOUKHE, Zijah BURZIĆ, Saša SAVIĆ, Slavica TERZIĆ, Danica SIMIĆ, Miodrag LISOV
}

\begin{abstract}
This paper presents a numerical study of the effect of main parameters (liner material, explosive charge, stand-off distance and the presence of the wave-shaper), on one hand, on the jet formation, jet velocity and the jet length, and on the other hand, on the penetration depth. We propose a numerical approach to evaluate their effects on the performance of the shaped charge. AUTODYN-2D software is used for numerical simulations of the shaped charge. The multi material Euler solver of the AUTODYN is used for the jet formation investigation, whereas, Lagrange solver is used for the resolution of the penetration problem. Results have shown that the presence of waveshaper increases the shaped charge depth penetration. For the standoff of 6 charge calibres, the jet penetration is found to be deepest. It is found that the jet velocity is proportional to the jet energy, and the penetration is proportional to the liner density. The oblique explosive reactive armor is more efficient for the protection of the main target. Numerical results have a good agreement with the data from available literature - with experimental results for the chosen explosives.
\end{abstract}

Keywords: ERA; HEAT; high explosive; metal liner; wave-shaper

\section{INTRODUCTION}

A shaped charge is generally a high explosive cylinder with a cavity in one end, lined with a thin metal layer and a detonator at the opposite end. Shaped charges are used to make a perforation, or a slender hole in a material that is otherwise difficult to penetrate [1]. Upon initiation, detonation of the explosive charge creates intense, focused, localized forces and a shock wave. The detonation wave propagates through the explosive charge and further impinges on the liner to the axis of symmetry [2]. The inner layer of material forms a high velocity jet, while the remaining material forms a low velocity slug [3]. The liner is usually conical, although it can have other axissymmetric geometries such as a hemisphere, tulip or trumpet, to name a few. Many liner materials have also been considered; examples include zirconium, steel and depleted uranium [1]. The shaped charge jets have excellent penetration capability into various targets. Thus, it has been successfully used in both military and civilian applications $[4,5]$. The penetration performance is very sensitive to the standoff degree where it decays rapidly if the latter is too large or too small. Slobodan Jaramaz et al [6] have validated their numerical model by investigating the influence of the stand-off distance and the apex cone angle on the penetration depth. Their results show that the jet can penetrate deeply for large standoff distance. Furthermore, they have found that the penetration is inversely proportional to apex cone angle. Liner material has also an effect on penetration [7]. The jet formation caused by the collapse of a shaped charge liner depends on the pressure delivered to the liner wall by the detonating explosive [8]. By optimizing explosive charge types, geometric configurations, initiation mode, and liner materials for shaped charge warhead, the jet penetration capability can be enhanced [3]. The penetrating effect of a shaped charge jet can be effectively weakened by fitting reactive armour elements on the outside of the armour itself. The reactive armour is composed of multi-layer elements - sandwich structure plates made of metal or a composite material and an intermediate explosive layer [9]. William P. et al. [10] investigated how to increase the tip velocity of a shaped charge by employing an air cavity wave shaper which overlaps the liner. This latter increases the tip velocity of the coherent portion of the liner to approximately $10 \mathrm{~km} / \mathrm{s}$. Nasser Dashtian Gerami et al. [11] studied the influence of the liner material on shaped charge penetration into thick concrete targets. Their results suggest that both copper and aluminium are capable to destroy thick concrete targets, but aluminium conical liner has more significant effect in creating penetration depth. Chang, B.H. et al. [12] enhanced the penetration depth for low density jet by $5.38 \%$ adding a certain fraction of copper powder in the matrix.

The problem with researches in the field of shaped charges is in the costs and time spent to carry out the experiments. Explosive charges and experimental perforation tests are expensive and take a lot of time to prepare and organize. In the process of a shaped charge warhead design, it is substantial to save money and efforts, and in the same time to have at disposal reliable data for effectiveness of different shaped charges configurations, for a variety of explosives, liner geometries and materials. For this reason it is useful to create a software solution - a model for simulation that gives results close enough to experimental ones, using already known input parameters, or for example, experimental results of some easy-tomeasure properties of explosives.

Various numerical codes have been developed in order to simulate the penetration process, with the aim to accurately represent the physical aspects of penetration whilst minimizing computational time. These codes have been developed and examined during past few decades, and some of them are PIESES code, HOCC code, PENJET, etc [2]. AUTODYN is one of these numerical models, a finite difference code which is used to model the stages of liner collapse, jet formation and jet penetration [5]. It is a commercially available software mostly used for analyzing high-velocity impacts, explosions, and shock waves, but also to analyze explosion welding [13]. There are also models using both a Lagrangian framework (e.g. "DYNA") and Eulerian formulation (e.g. "GRIM"), known as hydrocodes [1]. 
Series of numerical simulations performed with Autodyn-2D software will show the effect of different parameters into shaped charge jet formation, jet velocity and penetration capability and protection method for the main target using explosive reactive armour. The aim of these simulations is to save the time and resources needed for real field tests, and to provide a solid basis of data that could be used in practice, in future warhead design projects. The time and resources that will be saved, and the efficiency of the future warheads design process are the reason why the results obtained from this work are important. The experimental data used for the simulations in this paper were obtained in original experiments for different widely used explosives. Some of the observed explosives are used for a long time, based on TNT, while some are prominent, PBX explosives with polymer binder, belonging to the group of less sensitive explosives.

\section{NUMERICAL SIMULATION}

\subsection{Mesh Sensitivity}

In order to study the mesh sensitivity, five different meshes are utilized. The size of cells is selected to be 0.8 , $0.6,0.4,0.2$ and $0.1 \mathrm{~mm}$. Uniform and square cells are imposed in the jet formation region. The jet tip velocity for five different meshes are given in table 1 at $t=26 \mu \mathrm{s}$.

Table 1 Mesh sensitivity results
\begin{tabular}{|l|c|c|c|c|c|}
\hline Mesh size, mm & 0.8 & 0.6 & 0.4 & 0.2 & 0.1 \\
\hline Jet tip velocity, m/s & 5471 & 5704 & 6196 & 6308 & 6373 \\
\hline
\end{tabular}

Comparing with the results obtained in [14], the jet tip velocity is found to be in a good agreement using the square shape element of size $0.2 \mathrm{~mm}$. Thus, $0.2 \times 0.2 \mathrm{~mm}$ cell is used to simulate the jet formation of shaped charge taking into account a reasonable accuracy and time consumption. As the obtained results found in [15] are reliable, we utilized in our study the same mesh size of $0.5 \times 0.5 \mathrm{~mm}$ on the target used for the penetration problem.

\subsection{Numerical Setup}

AUTODYN software is used in this investigation. The multi-material Euler solver of AUTODYN is used to conduct the jet formation simulations. The Euler grid modelling is used to design the explosive, liner and waveshaper. The used mesh is $0.2 \times 0.2 \mathrm{~mm}$ as shown in Fig. 1 . Flow-out boundary condition is applied to all computational limits - expect the symmetry - to avoid effects of detonation reflections during propagation process [15]. The free space is filled with still air density of $0.001225 \mathrm{mg} / \mathrm{mm}^{3}$ and internal energy of $2.06640 \times 10^{5}$ micro-Joules.

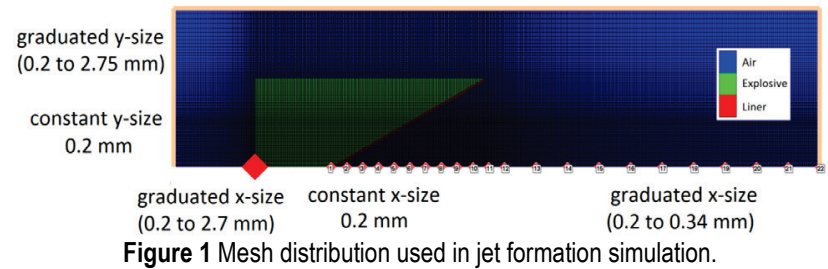

The shaped charge warhead used in this investigation is $56 \mathrm{~mm}$ in calibre and $73 \mathrm{~mm}$ in length. The cone angle of the liner is 60 with $1 \mathrm{~mm}$ of thickness. To measure the variation of the jet velocity in the axial direction, fixed gauge points are placed in the position shown in Fig. 1, this is also used to get the jet tip velocity with respect to time.

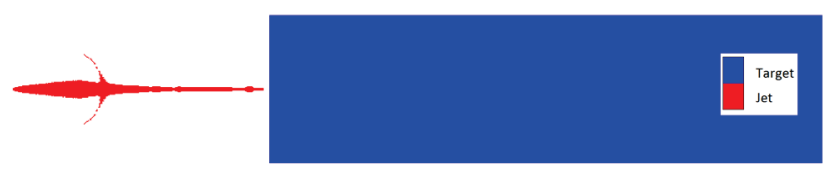

Figure 2 Penetration geometry problem

To simulate the penetration problem, the LAGRANGE solver of AUTODYN is used, in order to ensure the stability for the numerical computations [14]. The obtained results from Euler solver for jet formation are used for penetration problem using 'part fill' option. The initial geometry for the penetration problem is shown in Fig. 2. To ensure that the stability time-step remains at a reasonable level and solutions can continue to the desired termination time, the erosion option of AUTODYN is used. In AUTODYN, erosion is a numerical mechanism for the automatic removal (deletion) of elements during the simulation. The primary reason for using erosion is to remove too distorted elements from a simulation before becoming inverted (degenerate) [17]. Erosion strains of $200 \%$ and $600 \%$ were used for target and jet materials, respectively, according to [18]. The penetration is achieved when the jet is completely consumed or eroded on the crater walls, or when the jet velocity decays below a certain value, at which no change in the penetration is remarked with time.

\subsection{Material Models and Proprieties 2.3.1 Explosive}

Four military explosive compositions given in Tab. 2 have been studied numerically regarding the effect of explosive charge on the jet formation and penetration capability. The composition of explosive materials used in their study is cited below:

- FH-5: 95\% RDX/5\% wax - Montan wax (also known as lignite wax or OP wax),

- Heksotol: $90 \%$ RDX/10\% TNT,

- Okfol: $96.5 \% \mathrm{HMX} / 3.5 \%$ Montan wax,

- PBXN-5: 95\% HMX/5\% polymer - Viton A (fluoropolymer elastomer),

\section{Wherein:}

- RDX is high explosive hexogen (cyclotriamethylenetrinitramine);

- HMX is high explosive octogen (cyclotetramethylene-tetranitramine);

- $\quad$ TNT is high explosive trinitro-toluene;

- wax and polymer are inert phlegmatizers.

The explosive is simulated using the High Explosive Burn model along with Jones-Wilkins-Lee equation of state which controls the release of chemical energy [19]. The standard form of the JWL EOS is given by Lee et al. [20] in Eq. (1)

$$
P=A\left(1-\frac{\omega}{R_{1}}\right) e^{-R_{1} V}+B\left(1-\frac{\omega}{R_{2} V}\right) e^{-R_{2} V}+\frac{\omega E}{V}
$$


Wherein $P$ is the pressure and $A, B, R_{1}, R_{2}$ and $\omega$ are the JWL coefficients, $V$ is the relative volume and $E$ is the energy.

Those coefficients and detonation properties listed in Tab. 2 have been found experimentally by a cylinder test in Military Technical Institute in Belgrade.

\subsubsection{Liner}

The Shock-EOS and Steinberg-Guinan strength models are used to describe the deformation of liner [21]. The liner parameters for the Shock-EOS and SteinbergGuinan models are listed in Tab. 3.

Table 2 The JWL coefficients and the detonation proprieties

\begin{tabular}{|l|c|c|c|c|c|c|c|c|c|}
\hline \multicolumn{1}{|c|}{ Explosive } & $A, \mathrm{kPa}$ & $B, \mathrm{kPa}$ & $R_{1}$ & $R_{2}$ & $\omega$ & $\rho, \mathrm{g} / \mathrm{cm}^{3}$ & $D, \mathrm{~m} / \mathrm{s}$ & $P, \mathrm{kPa}$ & $E / \mathrm{Unit} \mathrm{volume}, \mathrm{kJ} / \mathrm{m}^{3}$ \\
\hline RDX [9] & $5.81 \times 10^{8}$ & $6.80 \times 10^{6}$ & 4.1 & 1 & 0.1 & 1.70 & 8390 & $3 \times 10^{7}$ & $9.00 \times 10^{6}$ \\
\hline FH-5 & $1.173 \times 10^{8}$ & $3.43 \times 10^{6}$ & 5.3 & 0.7 & 0.3 & 1.60 & 7930 & $2.42 \times 10^{7}$ & $8.71 \times 10^{6}$ \\
\hline Heksotol & $5.70 \times 10^{8}$ & $8.89 \times 10^{6}$ & 4.4 & 1.5 & 0.095 & 1.59 & 7820 & $2.45 \times 10^{7}$ & $8.49 \times 10^{6}$ \\
\hline Okfol & $6.82 \times 10^{8}$ & $7.27 \times 10^{6}$ & 4.4 & 0.92 & 0.07 & 1.68 & 8211 & $2.9 \times 10^{7}$ & \\
\hline PBXN-5 & $7.24 \times 10^{8}$ & $3.95 \times 10^{6}$ & 4.1 & 0.7 & 0.04 & 1.84 & 8826 & $3.7 \times 10^{7}$ & $9.88 \times 10^{6}$ \\
\hline
\end{tabular}

Table 3 The Shock EOS and the Steinberg-Guinan strength model

\begin{tabular}{|l|c|c|c|c|c|c|c|c|c|c|c|}
\hline \multicolumn{1}{|c|}{ Liner } & $\rho, \mathrm{g} / \mathrm{cm}^{3}$ & $\begin{array}{c}\text { Gruneisen } \\
\text { coefficient }\end{array}$ & $C_{1}$ & $S_{1}$ & $T_{\text {ref }}, \mathrm{K}$ & $Y, \mathrm{kPa}$ & $\begin{array}{c}\text { Shear modulus, } \\
\mathrm{kPa}\end{array}$ & $Y_{\max }, \mathrm{kPa}$ & $B$ & $n$ & $T_{\operatorname{melt}}, \mathrm{K}$ \\
\hline Copper & 8.93 & 2.02 & $3.94 \times 10^{3}$ & 1.49 & 300 & $1.2 \times 10^{5}$ & $4.77 \times 10^{7}$ & $6.4 \times 10^{5}$ & 36 & 0.45 & 1790 \\
\hline Aluminium & 2.78 & 2.00 & $5.32 \times 10^{3}$ & 1.34 & 300 & $2.6 \times 10^{5}$ & $2.86 \times 10^{7}$ & $7.6 \times 10^{5}$ & 310 & 0.18 & 1220 \\
\hline Tungsten & 19.29 & 1.67 & $4.03 \times 10^{3}$ & 1.24 & 300 & $2.2 \times 10^{6}$ & $1.60 \times 10^{8}$ & $4.0 \times 10^{6}$ & 7.7 & 0.13 & 4520 \\
\hline Platinum & 21.44 & 2.74 & $3.64 \times 10^{3}$ & 1.54 & 300 & $3.0 \times 10^{4}$ & $6.37 \times 10^{7}$ & $3.4 \times 10^{5}$ & 1300 & 0.19 & 2840 \\
\hline
\end{tabular}

\section{RESULTS AND DISCUSSIONS}

\subsection{Numerical Verification}

In order to verify our numerical model, a series of numerical simulations have been carried out. Obtained results were compared with the experimental data from [14]. RDX was used as explosive charge, copper as liner material and steel as target. A comparison of numerical and experimental results is illustrated in Tab. 4 in the moment when the jet hits the target.

The experimental results [14], shown in Tab. 4, were used to verify the numerical model. The jet tip velocities obtained from the numerical simulation and in the experiment were respectively $6301 \mathrm{~m} / \mathrm{s}$ and $6520 \mathrm{~m} / \mathrm{s}$ with an error less than $3.5 \%$. In addition, the difference between the penetration depth in the experiment $(209.80 \mathrm{~mm})$ and simulation $(241.20 \mathrm{~mm})$ is about $13 \%$. Consequently, good agreement was observed.

Table 4 Comparison between numerical results and results from [14]

\begin{tabular}{|l|c|c|}
\hline & Jet velocity, $\mathrm{m} / \mathrm{s}$ & Penetration, $\mathrm{mm}$ \\
\hline Autodyn & 6301 & 209.80 \\
\hline Reference [14] & 6520 & 241.20 \\
\hline
\end{tabular}

\subsection{Effect of the Main Parameters \\ 3.2.1 Effect of the Explosive Charge}

In this part of simulation, the effect of the explosive charge on the jet behaviour, formation and penetration depth is investigated. Fig. 3 and Fig. 4 show the axial velocity measured by gauges points and the velocity distribution on the jet at stand-off of 2 charge calibre respectively.

The results show that the jet tip velocity has almost the same behaviour for all explosives. It is observed that the velocity increases until a maximum value. After that, the jet tip velocity decreases slightly before it stabilizes at a constant value. Tab. 5 shows the results obtained by numerical simulation for different explosive charges.
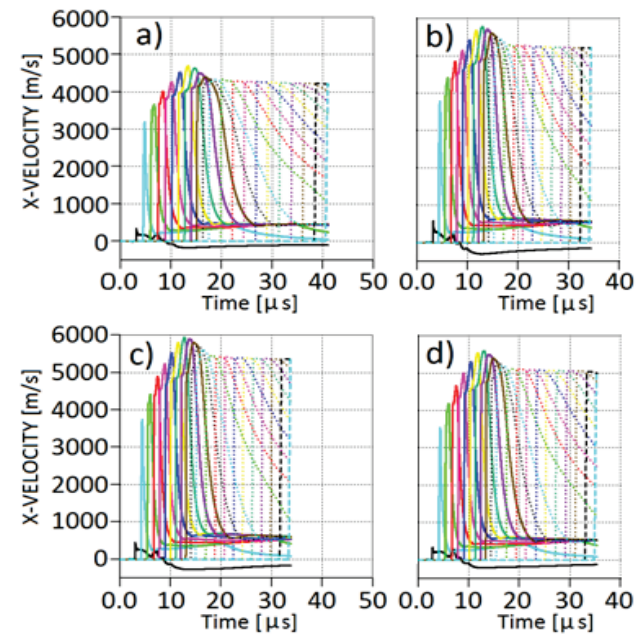

Figure 3 Variation of the axial velocity for different types of explosive: a) FH-5; b) Heksotol; c) Okfol; d) PBXN-5

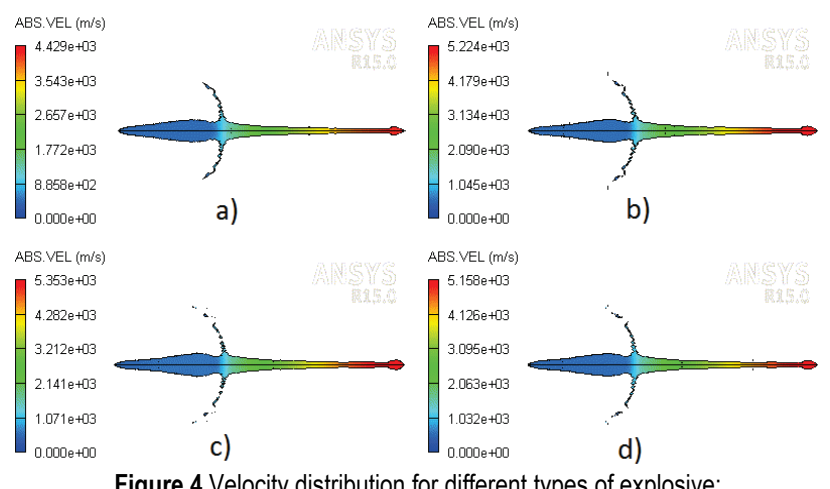

Figure 4 Velocity distribution for different types of explosive:

a) FH-5; b) Heksotol; c) Okfol; d) PBXN-5.

From the results, the jet is faster for shaped charge with explosive Okfol because of the energy obtained from the high explosive during its detonation is related to Gurney velocity of this explosive, which is the energy released from the high explosive and transformed into mechanical work, imparted the liner element. Gurney velocity increases with the detonation velocity and/or the detonation pressure of the explosive which leads to the increase of the jet tip velocity. As a result, the jet kinetic 
energy and its penetration potential into target will be enhanced.

Table 5 Simulation results for different pressed explosive charges

\begin{tabular}{|l|c|c|c|c|}
\hline Explosive material & FH-5 & Heksotol & Okfol & PBXN-5 \\
\hline Impact time $\mu \mathrm{s}$ & 41.05 & 34.46 & 33.80 & 35.31 \\
\hline Impact velocity m/s & 4429 & 5224 & 5353 & 5158 \\
\hline Jet length mm & 75.17 & 84.75 & 86.04 & 83.11 \\
\hline Penetration $\mathrm{mm}$ & 116.70 & 161.25 & 194.22 & 169.25 \\
\hline Hole radius $\mathrm{mm}$ & 5.90 & 7.80 & 8.30 & 7.77 \\
\hline
\end{tabular}

\subsubsection{Effect of the Liner Material}

Numerical simulations were carried out on four different liner materials in order to determine their effect on the jet formation and penetration capability. Fig. 5 and Fig. 6 show the velocity distribution in the shaped charge jet and the axial velocity profile for each liner material when the jet hits the target respectively.
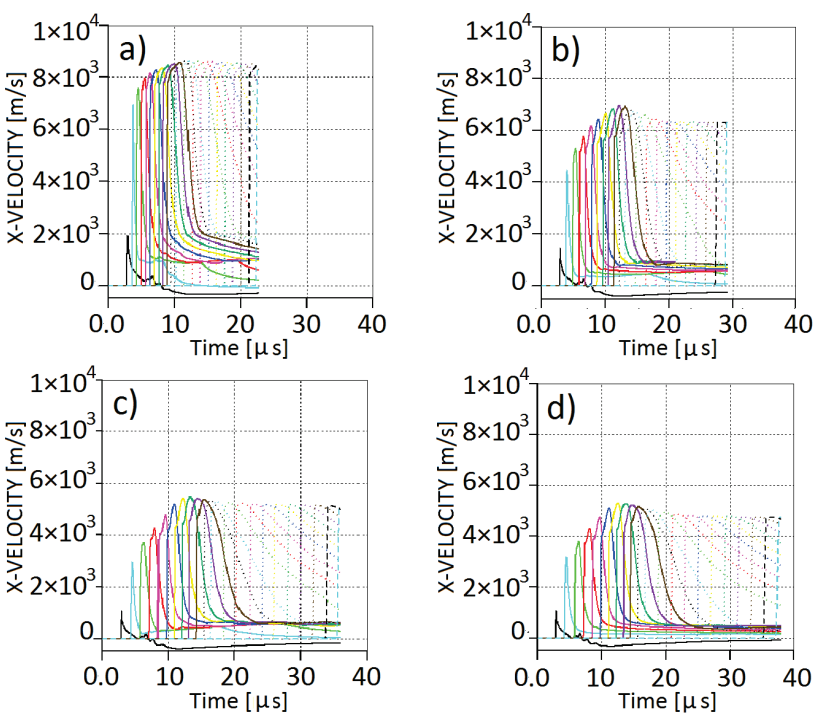

Figure 5 Variation of the axial velocity for different types of liner material: a) Aluminium; b) Copper; c) Tungsten; d) Platinum.
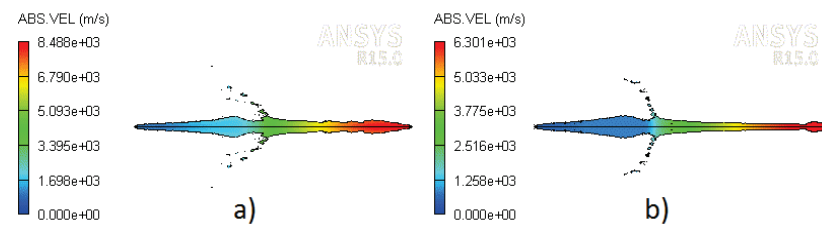

a)

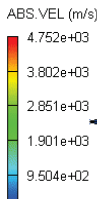

c)

d) penetration capability. The reason for this is the high density of platinum jet.

Table 6 Simulation results for different type of liner

\begin{tabular}{|l|c|c|c|c|}
\hline \multicolumn{1}{|c|}{ Explosive material } & Aluminium & Copper & Tungsten & Platinum \\
\hline Impact time, $\mu \mathrm{s}$ & 22.82 & 29.22 & 36.07 & 37.92 \\
\hline Impact velocity, m/s & 8488 & 6301 & 5131 & 4752 \\
\hline Jet length, mm & 77.20 & 86.64 & 96.74 & 97.02 \\
\hline Penetration, $\mathrm{mm}$ & 129.30 & 209.80 & 261.50 & 321.21 \\
\hline Hole radius, $\mathrm{mm}$ & 13.20 & 9.30 & 4.50 & 8.40 \\
\hline
\end{tabular}

\subsubsection{Effect of the Standoff Distance}

In this part, we investigate the penetration problem with different stand-off distance. The target is fixed at 0,2 , 4, 6, 8, 10 and 12 charge calibre from the base of the liner. RDX and copper are used as explosive and liner respectively.

Fig. 7 shows the variation of the penetration depth with stand-off distance.

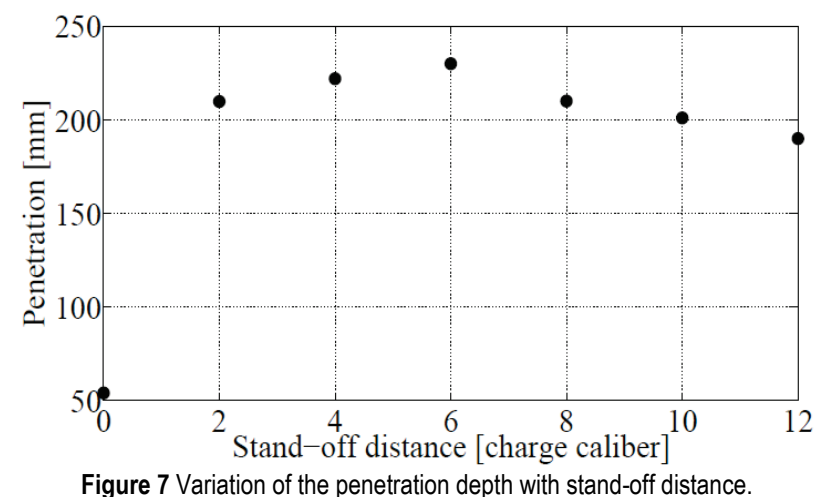

Results show that the penetration phenomena divided into two stages; the first is when we increase the stand-off distance from zero to 6 charge calibres. At this stage, the penetration increases from $54 \mathrm{~mm}$ to reach her maximum in 6 charge calibres stand-off distance. After this stage, the jet starts to lose energy and becomes weaker which explains the fact that the penetration decreases even when the stand-off distance increases.

\subsubsection{Effect of the Wave-shaper}

In order to determine the influence of the wave-shaper on jet formation and penetration, the shaped charge is simulated together with wave-shaper as shown in Fig. 8. Teflon was used as wave-shaper material with density of $2.15 \mathrm{~g} / \mathrm{cm}^{3}$, with RDX as explosive and copper as liner.
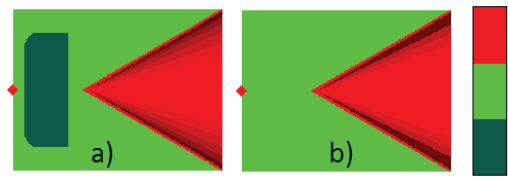

Liner

Figure 6 Variation distribution for different types of liner material: a) Aluminium b) Copper; c) Tungsten; d) Platinum.

The results show that the jet tip velocity has almost the same behaviour for all liner materials. It is observed that the velocity increases until a maximum value. After that, the jet tip velocity decreases slightly before it stabilizes at a constant value. Tab. 6 shows the numerical results for different liner material.

The results show that the Aluminium jet has the highest velocity with the poorest penetration depth. Platinum jet has the smallest jet velocity but the strongest
Figure 8 Shaped charge warhead type. a) with wave-shaper b) without waveshaper.

Fig. 9 shows the axial velocity profile for the shaped charge jet with and without wave-shaper.

Results illustrate that the jet tip velocity for shaped charge without wave-shaper is $6291 \mathrm{~m} / \mathrm{s}$, and with waveshaper is $10100 \mathrm{~m} / \mathrm{s}$, with the leading hypervelocity particles travelling much faster as shown in Fig. 9. That 
happens because the wave-shaper causes the smallest incidence inclination angle as shown in Fig. 10. As it will increase the collapse velocity of the liner, the jet velocity will be increased.

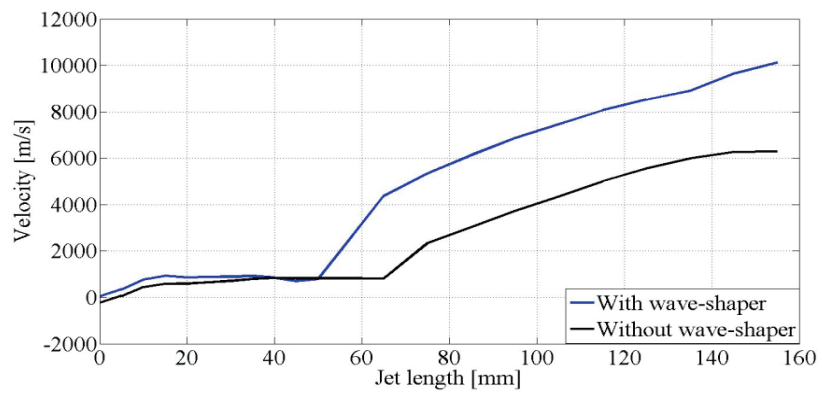

Figure 9 The axial velocity profile: a) with wave-shaper; b) without wave-shaper. Variation of the penetration depth with stand-off distance. a)

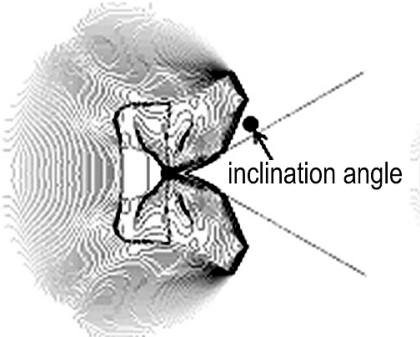

b)

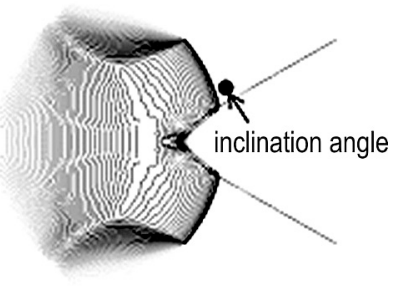

Figure 10 The shape of detonation wave: a) with wave-shaper; b) without waveshaper.

The Tab. 7 shows results of the effect of the waveshaper on jet formation and penetration capability.

Table 7 Simulation results for the effect of the wave-shape

\begin{tabular}{|l|c|c|}
\hline \multicolumn{1}{|c|}{ Warhead type } & Without wave-shaper & With wave-shaper \\
\hline Impact time, $\mu \mathrm{s}$ & 29.22 & 21.68 \\
\hline Impact velocity, $\mathrm{m} / \mathrm{s}$ & 6301 & 10130 \\
\hline Jet length, $\mathrm{mm}$ & 86.64 & 106.15 \\
\hline Penetration, $\mathrm{mm}$ & 209.80 & 250.01 \\
\hline
\end{tabular}

From the results it may be observed that the waveshaper has effect on jet velocity and length. The presence of the wave-shaper on shaped charge warhead causes the increase of the jet velocity and jet length. As a result, the wave-shaper can enhance the penetration capability of the shaped charge jet.

\subsection{Effect of the Explosive Reactive Armor to Disturb the Shaped Charge Jet}

In this part the explosive reactive armour was used to identify this influence on the penetration depth. Explosive reactive armour was formed from 2 plates of steel backed by explosive charge, TNT. In this investigation both the front plate of explosive reactive armour and interlayer explosive were $8 \mathrm{~mm}$ of thickness, the rear plate was $2 \mathrm{~mm}$ of thickness. The explosive reactive armour was inclined for $25,35,45,55$ and $65^{\circ}$ to identify the effect of the attack angle, Fig. 11. RDX and copper were used as explosive and liner respectively in jet formation simulation.

Tab. 8 shows the penetration results for the effect of the explosive reactive armour and the attack angle on the disturbation of the shaped charge jet.

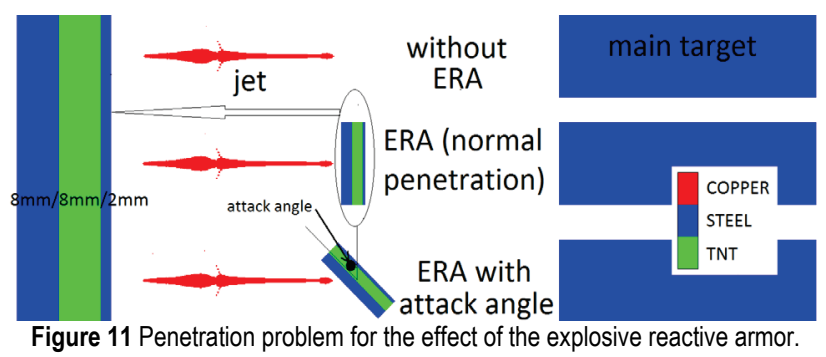

Table 8 Simulation results for the effect of the wave-shaper

\begin{tabular}{|l|c|c|c|c|c|c|}
\hline Attack angle $^{\circ}$ & 0 & 25 & 35 & 45 & 55 & 65 \\
\hline Penetration, $\mathrm{mm}$ & 182.2 & 82.00 & 81.50 & 71.00 & 64.95 & 66.20 \\
\hline
\end{tabular}

Results indicate that the normal penetration in main target with normal impact in the explosive reactive armour is reduced by $20 \%$ to $182.2 \mathrm{~mm}$.

The penetration in main target is reduced significantly using explosive reactive armour with an attack angle. The minimal residual penetration is $64.9 \mathrm{~mm}$ for attack angle of $55^{\circ}$. This has happened because the front plate moved and cut the jet after detonation of explosive reactive armour. This means that the penetration will be disturbed by the formation of more jet segments.

\section{CONCLUSION}

The study presented in this paper shows the influence of different parameters on the shaped charge jet formation and penetration depth on one hand, and the effect of the explosive reactive armour and its attack angle to disturb the shaped charge jet on the other hand. The main outcomes of this study are as follows:

1) The required explosive for shaped charges must have high velocity of detonation and high density to provide a significant detonation pressure. The latter results in fast jet tip velocity and deeper penetration. It is found that PBXN5 is the most effective explosive contrary to the FH-5 which was observed to be the weakest one.

2) Since the penetration is proportional to the liner density, the platinum liner has the deepest penetration, and the aluminium's penetration is the poorest.

3) The penetration depth reaches its maximal value in the stand-off of 6 charge calibre.

4) The penetration capability of the shaped charge could be enhanced using the wave-shaper.

5) The explosive reactive armour with inclination of $55^{\circ}$ provides much better protection.

\section{REFERENCES}

[1] Chris, P. (2005). Penetration of Shaped Charge. PhD Thesis, Corpus Christo College, University of Oxford.

[2] Himanshu, S. (2012). Theoretical Modelling of Shaped Charge in the Last Two Decades (1990-2010). A Review. Central European of Energetic Materials, 9(2), 155-185.

[3] Wang, C., Ding, J. \& Zhao, H. (2015). Numerical simulation on jet formation of shaped charge with different liner materials. Defence Science Journal, 65(4), 279-286. https://doi.org/10.14429/dsj.65.8648

[4] Elshenawy, T., Elbeih, A., \& Ming Li, Q. (2016). A modified penetration model for copper-tungsten shaped charge jets with non-uniform density distribution. Central European Journal of Energetic Materials, 13(4), 927-943. https://doi.org/10.22211/cejem/65141 
[5] Elshenawy, T. (2012). Criteria of Design Improvement of Shaped Charges Used as Oil Well Perforators. PhD Thesis, University of Manchester, Faculty of Engineering and Physical Sciences, School of Mechanical, Aerospace and Civil Engineering.

[6] Micković, D., Jaramaz, S., Elek, P., Jaramaz, D., \& Micković, D. (2012). A Model for Shaped Charge Warhead Design. Strojniški vestnik - Journal of Mechanical Enginering, 58(6), 403-410. https://doi.org/10.5545/sv-jme.2012.335

[7] Carlucci, D. E. \& Jacobson, S. S. (2007). Ballistics - Theory and design of guns and ammunition, Taylor \& Francis Group, LLC. New York. https://doi.org/10.1201/9781420066197

[8] Simon, J. (1974). The effect of explosive detonation characteristics on shaped charge performance. Ballistic Research Laboratories - BRL, Report No. AD-785 680, 98112.

[9] Cudzilo, S., Dyjak, S., \& Trzcinski, W. A. (2012). Preparation and characterization of monolithic nitrocellulose-cellulose composites. Central European Journal of Energetic Materials, 9(2), 139-146.

[10] Walters, W. P. \& Scheffler. D. R. (2007). A method to increase the tip velocity of a shaped charge jet. $23^{r d}$ International Symposium on Ballistics. https://doi.org/10.2495/CBAL070091

[11] Gerami, N. D., Liaghat, G. H., Rahimi, G. H., Moghadas, S \& Khazraiyan, N. (2017). Analysis of liner effect on shaped charge penetration into thick concrete targets. The Brazilian Society of Mechanical Sciences and Engineering, 39, 31893201. https://doi.org/10.1007/s40430-017-0797-6

[12] Chang, B. H., Yin, J. P. Cui, Z. Q., \& Liu, T. X. (2015). Numerical simulation of modified low-density jet penetrating shell charge. International Journal of Simulation Modelling, 14(3), 426-437. https://doi.org/10.2507/IJSIMM14(3)5.295

[13] Li, Y., Liu, C., Yu, H., Zhao, F. \& Wu, Z. (2017). Numerical Simulation of Ti/Al Bimetal Composite Fabricated by Explosive Welding. Metals, 7(10), 407, 1-13. https://doi.org/10.3390/met7100407

[14] Xiang-Dong, L. Yan-Shi, Y., \& Sheng-Tao, L. (2014). A Numerical Study on the Disturbance of Explosive Reactive Armors to Jet Penetration. Defence Technology, 10, 66-75. https://doi.org/10.1016/j.dt.2014.01.006

[15] Elshenawy, T. \& Li, Q. M. (2013). Influence of target strength and confinement on the penetration depth of an oil well perforator. International Journal of Impact Engineering, 54, 130-137. https://doi.org/10.1016/j.jijmpeng.2012.10.010

[16] Marković, M. D., Milinović, M. P., Jeremić, O. M., \& Jaramaz, S. S. (2016). Simulation of changes in temperature and pressure fields during high speed projectiles forming by explosion. Thermal Science, 20(5), 1741-1752. https://doi.org/10.2298/TSCI151217073M

[17] AUTODYN. (1998). Theory Manual, Revision 4.0, Century Dynamics Inc.

[18] Berg, V. S. \& Preece, D. S. (2004). Shaped Charge Induced Concrete Damage Predictions Using RHT Constitutive Modelling. Journal of International Society of Explosives Engineers, 2, 261-272.

[19] Downes, D., Bouamoul, A., \& Nsan, M. N. (2014). Numerical simulation of the shaped charge, Defence Research and Development. Report No. DRDC-RDDC2014-P38

[20] Lee, L. Hornig, C., \& Kury, W. (1968). Adiabatic expansion of high explosive detonation products. Lawrence Radiation Laboratory. Report No. UCRL-50422 https://doi.org/10.2172/4783904
[21] Zhe, W., Jian, J., Shu, W., \& Han, L. (2017). Jet Formation and Penetration Study of Double-layer Shaped Charge. Journal of Energetic Materials, 36(2), 1-17. https://doi.org/10.1080/07370652.2017.1326987

[22] Džingalašević, V. (2008). Odredđivanje Brizantnosti Eksploziva Cilindar Testom, Military Technical Institute, Report No. VTI -04-01-0533, Belgarde.

\section{Contact information:}

\section{Hicham KEMMOUKHE}

University of Defence, Military Academy,

Pavla Jurišića Šturma 30, 11000 Belgrade, Serbia

hkemmoukhe@gmail.com

\section{Zijah BURZIĆ}

Military Technical Institute

Ratka Resanovića 1, 11030 Belgrade, Serbia

zijah.burzic@vti.vs.rs

\section{Saša SAVIĆ}

Military Technical Institute,

Ratka Resanovića 1, 11030 Belgrade, Serbia

svsavic@hotmail.com

\section{Slavica TERZIĆ}

Military Technical Institute,

Ratka Resanovića 1, 11030 Belgrade, Serbia;

slavica@algodesk.com

\section{Danica SIMIĆ}

Corresponding author

Military Technical Institute,

Ratka Resanovića 1, 11030 Belgrade, Serbia

simic_danica@yahoo.com

\section{Miodrag LISOV}

Military Technical Institute,

Ratka Resanovića 1, 11030 Belgrade, Serbia vti@mod.gov.rs 